\title{
The conservation value of unlogged and logged forests for native mammals on the East Coast of Peninsular Malaysia
}

\begin{abstract}
Tropical forests across the world provide important habitats for a diverse number of conservation priority species, yet are under threat from a range of anthropogenic impacts including logging. This study aims to quantify mammalian biodiversity in unlogged and logged forests in the adjoining Tembat and Petuang Forest Reserves, Terengganu, on the East Coast of Peninsular Malaysia. Data was collected over a series of surveys using direct and indirect observation methods from 2008 to 2014. A total of 30 medium and large sized mammals species were identified, with 27 of those species found in unlogged forests and 22 species in logged forests. Carnivores encompassed 11 species from 67 observations representing $15 \%$ of the total number of observations. The family Felidae had the highest number of species (six species), followed by Hylobatidae, Cercopithecidae and Suidae with three species each. A total of 17 species contributed to more than $90 \%$ of the mammal community in the unlogged and logged forests, while six species were uncommon and only observed once during the entire survey. Species abundance in the unlogged forest was significantly greater than the logged forests, but the difference was not significant for species richness. This study provides critical baseline information on the impact of unlogged and logged forests and the identification of threatened species warrant the establishment of conservation measures such as anti-poaching patrol and ranger stations in the study area.
\end{abstract}

Keyword: Biodiversity; Forest reserve; Logging; Mammals; Species composition 